\title{
Trimeric plasmonic molecules: the role of symmetry
}

\author{
Lev Chuntonov and Gilad Haran
}

Department of Chemical Physics, Weizmann Institute of Science, Rehovot 76100, Israel

\section{SUPPORTING INFORMATION}

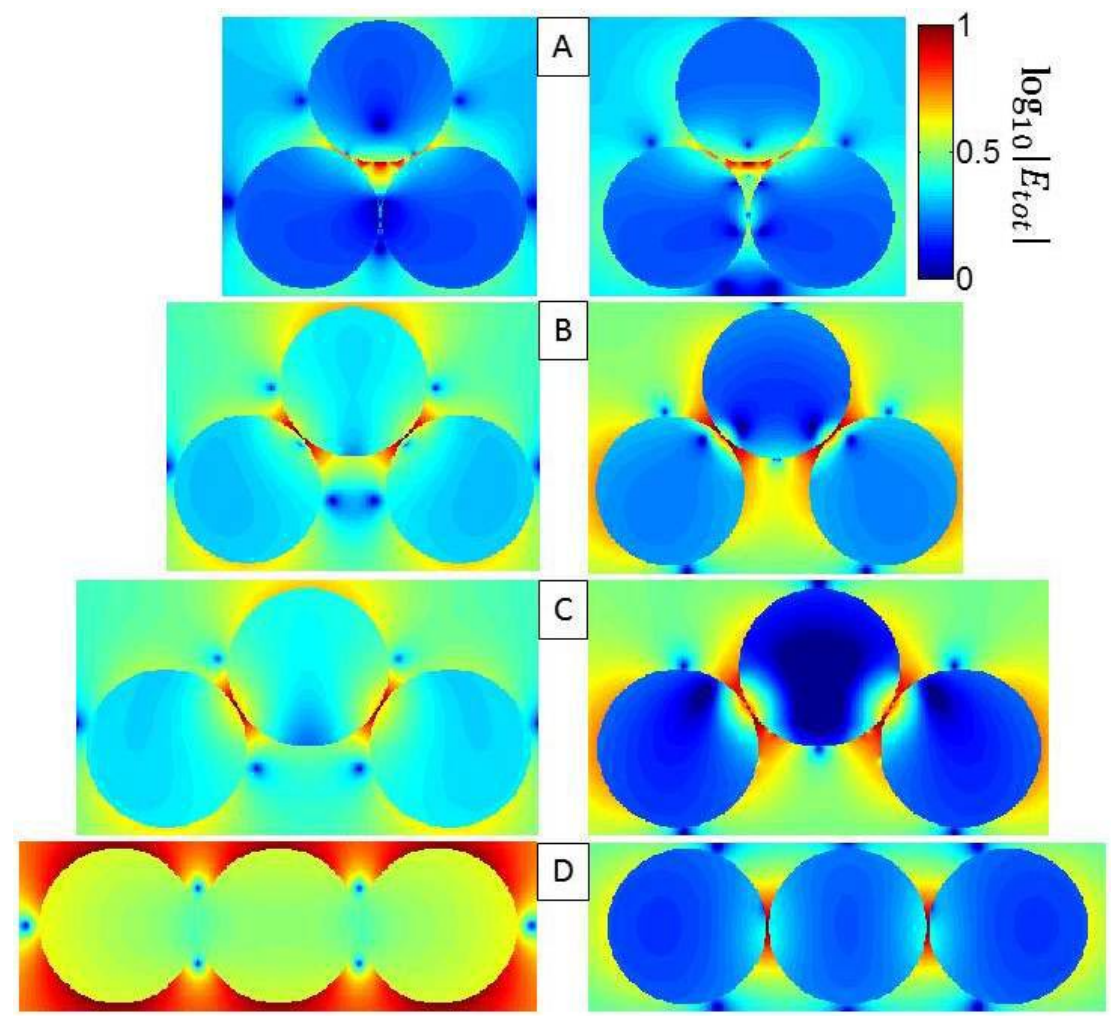

Figure S1. Simulated electric field plots corresponding to the charge plots shown in Fig. 1. The field magnitude is normalized to its maximal value in each case. Left column - transverse polarization of excitation light, right column - longitudinal polarization of excitation light. The plots are simulated for resonant excitation at: panel A - $645 \mathrm{~nm}$ (left), $645 \mathrm{~nm}$ (right); panel B - $625 \mathrm{~nm}$ (left), $710 \mathrm{~nm}$ (right); panel C - $610 \mathrm{~nm}$ (left), $725 \mathrm{~nm}$ (right); panel D - 600 $\mathrm{nm}$ (left), $745 \mathrm{~nm}$ (right). Note the logarithmic scale of the color code. Interestingly, for excitation of the $D_{3 \mathrm{~h}}$ symmetry cluster with both transverse and longitudinal light polarization (panel A), only two out of three gaps between the nanoparticles are "hot". This is a result of the mode structure of the plasmon excitation discussed in the paper. Obviously, a different pair of gaps may be selected by rotating the light polarization by $2 \pi / 3$. 